\title{
The continued debate on solar coronal EUV waves
}

\author{
PengFei Chen* \\ School of Astronomy E Space Science, Nanjing University, Nanjing 210023, China
}

Received October 9, 2016; accepted November 9, 2016; published online November 28, 2016

Citation: P. F. Chen, The continued debate on solar coronal EUV waves, Sci. China-Phys. Mech. Astron. 60, 029631 (2017), doi: 10.1007/s11433-016-0397-0

Gravity is everywhere in the universe [1,2], and the same is true of the magnetic field [3-5]. Similar to gravity, magnetic field also controls the dynamics of the matter in the universe, over $99.9 \%$ of which is in the plasma state. Somewhat different from gravity which becomes overwhelming in large scales, magnetic field becomes more important in smaller scales. This is probably why magnetic field has not been considered too seriously in and above the galactic scale, whereas it becomes more and more important in the stellar scale.

Our Sun is a star close to us, and is the only star which offers crucial light and heat for humans to survive on the Earth. To the naked eyes, the Sun is a constantly shining star. However, it is actually full of dynamics and even violent activity [6], and all of these are owed to magnetic field [7], without which the Sun would be a boring object hanging in the sky.

The magnetic field of the Sun is thought to originate from the bottom of the convection zone, roughly at a radius of $0.7 R_{\odot}$, where differential rotation at different latitudes stretches and amplifies any initial tiny seed magnetic field through a dynamo process [8]. When the magnetic field is amplified to $\sim 10^{5} \mathrm{G}$, it becomes buoyantly unstable, and rises to the solar surface, forming sunspots in pairs. With the effects of surface motions and magnetic diffusion, the magnetic field fills in almost the whole atmosphere, i.e., part of the photosphere, most of the chromosphere, and all over the extended corona, which is heated by the magnetic field to 1-2 MK [9]. Because of the high optical thickness, the thin photosphere is so far the only layer of the solar atmosphere where magnetic field can be measured reliably. While efforts have been

* Corresponding author (email: chenpf@nju.edu.cn) made to diagnose the magnetic field in the chromosphere and the corona with the conventional method based on Zeeman effect, several indirect approaches have been proposed [10], one of which is the coronal seismology [11], which is based on waves propagating in the corona.

In the solar corona, there are various categories of waves. The most studied waves are standing magnetohydrodynamic (MHD) waves trapped in coronal loops. By measuring the oscillation period of a loop, the local magnetic field can be estimated [12]. However, the limitation of this method is that we can only derive the magnetic field averaged along the localized coronal loop, with the magnetic field of the vast corona outside the loop remaining unknown. Fortunately, a global wave phenomenon was discovered in the corona in 1997 as shown in Figure 1 [13], which ushers a new era for global coronal seismology. Because this wave phenomenon was discovered using the observations of the Extreme ultraviolet Imaging Telescope (EIT) on board the Solar and Heliospheric Observatory ( $\mathrm{SOHO}$ ) satellite, it was initially christened coronal "EIT waves". When reported in the news (including some Chinese news media) by the journalists, it was called "solar tsunami". While the nature of the terrestrial tsunami is well known, the nature of the coronal "EIT waves" has been controversial, and Chinese colleagues have been actively participating in the debate, both from observational [14-34] and modeling [35-42] points of view.

Initially, "EIT waves" were thought to be fast-mode MHD waves [43-45]. However, when several stationary "EIT wave" fronts were found to exist at magnetic separatrices, Delannee [46] started to challenge the wave model since apparently there is no reason for a fast-mode wave to stop at magnetic separatrices, where magnetic connectivity, not 

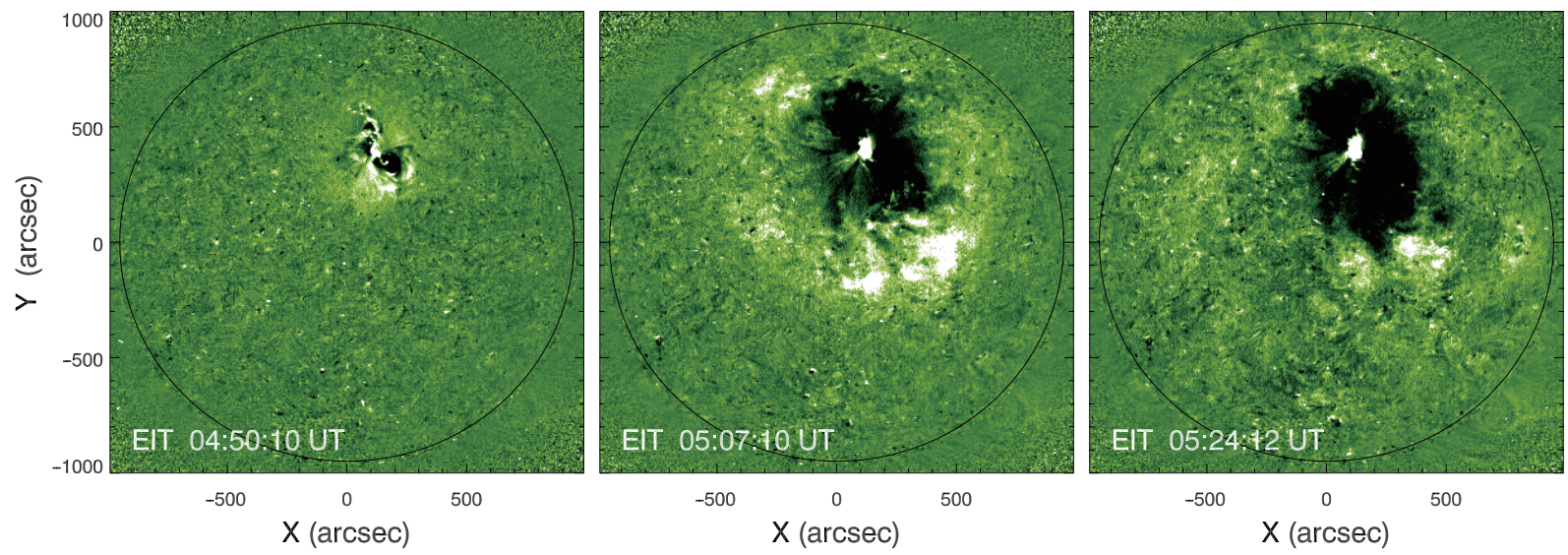

Figure 1 (Color online) Evolution of the coronal "EIT wave" observed on 1997 May 12, which was analyzed by ref. [13], marking the discovery of "EIT waves" (from ref. [6]).

the magnetic strength, changes drastically. Inspired by their questioning, various alternative models have been proposed since then. For example, the magnetic fieldline stretching model [35] can not only explain why "EIT waves" stop at magnetic separatrices, but also predicted that once the observational cadence is high enough, we should be able to detect two coronal waves in extreme ultraviolet (EUV) wavelengths, with the faster one being a fast-mode MHD wave (or shock wave) and the slower one being the conventional "EIT wave", which is an apparent propagation of brightenings generated by magnetic fieldline stretching. Other models, including the slow-mode wave model $[39,41]$, can explain some other observational features.

With the discovery of two clearly distinguished coronal waves [47-51], it was apparent to me that, as we predicted in 2002 [35], there are two types of coronal EUV waves, which have different velocities and different formation mechanisms [52]. This is why I wrote a review paper titled "A Converging View on EIT Waves" [53]. However, even until now, some colleagues insist that coronal "EIT waves" are fast-mode MHD waves. From 2014 to 2016, I was involved in an international "EIT wave" consortium, and we organized 3 meetings in the International Space Science Institute (ISSI) in Bern. Most of the consortium members, except me, also tend to think that coronal "EIT waves" are fast-mode MHD waves. Therefore, it seems that the debate will continue.

Looking back, it is seen that all of the efforts challenging the initial wave model for coronal "EIT waves" started with the discovery of the stationary EUV wave fronts near magnetic separatrices [46]. Ironically, recently we performed numerical simulations in order to explain the stationary EUV wave front observed by Chandra et al. [54]. We proposed that this type of stationary EUV waves are slow-mode MHD waves converted from fast-mode MHD waves near the place where the Alfvén speed is equal to the sound speed [55]. The converted slow-mode wave cannot propagate across magnetic field lines, leading to a stationary front.
This is how science goes. It often, if not always, develops in such a spiral way.

This work was supported by the National Natural Science Foundation of China (Grant Nos. 11533005, and 11025314), and Jiangsu 333 Project.

1 D. Blair, L. Ju, C. N. Zhao, L. Q. Wen, Q. Chu, Q. Fang, R. G. Cai, J. R. Gao, X. C. Lin, D. Liu, L. A. Wu, Z. H. Zhu, D. H. Reitze, K. Arai, F. Zhang, R. Flaminio, X. J. Zhu, G. Hobbs, R. N. Manchester, R. M. Shannon, C. Baccigalupi, W. Gao, P. Xu, X. Bian, Z. J. Cao, Z. J. Chang, P. Dong, X. F. Gong, S. L. Huang, P. Ju, Z. R. Luo, L. E. Qiang, W. L. Tang, X. Y. Wan, Y. Wang, S. N. Xu, Y. L. Zang, H. P. Zhang, Y. K. Lau, and W. T. Ni, Sci. China-Phys. Mech. Astron. 58, 120402 (2015).

2 Z. J. Cao, Sci. China-Phys. Mech. Astron. 59, 110431 (2016).

3 G. J. Cao, X. Zhou, and N. Wang, Sci. China-Phys. Mech. Astron. 58, 039502 (2015).

4 W. W. Na, G. F. Lin, and R. Q. Huang, Sci. China-Phys. Mech. Astron. 58, 029501 (2015)

5 H. Tong, Sci. China-Phys. Mech. Astron. 59, 619501 (2016).

6 P. F. Chen, Living Rev. Solar Phys. 8, 1 (2011).

7 J. X. Wang, Y. Z. Zhang, H. He, A. Q. Chen, C. L. Jin, and G. P. Zhou, Sci. China-Phys. Mech. Astron. 58, 599601 (2015).

8 A. R. Choudhuri, Sci. China-Phys. Mech. Astron. 60, 019601 (2017).

9 B. C. Low, Sci. China-Phys. Mech. Astron. 58, 015201 (2015).

10 P. F. Chen, Sci. China Ser. G-Phys. Mech. Astron. 52, 1785 (2009).

11 V. M. Nakariakov, and E. Verwichte, Living Rev. Solar Phys. 2, 3 (2005).

12 Y. Guo, R. Erdélyi, A. K. Srivastava, Q. Hao, X. Cheng, P. F. Chen, M. D. Ding, and B. N. Dwivedi, Astrophys. J. 799, 151 (2015).

13 B. J. Thompson, S. P. Plunkett, J. B. Gurman, J. S. Newmark, O. C. St. Cyr, and D. J. Michels, Geophys. Res. Lett. 25, 2465 (1998).

14 P. F. Chen, Astrophys. J. 641, L153 (2006).

15 P. F. Chen, Astrophys. J. 698, L112 (2009).

16 S. Ma, M. J. Wills-Davey, J. Lin, P. F. Chen, G. D. R. Attrill, H. Chen, S. Zhao, Q. Li, and L. Golub, Astrophys. J. 707, 503 (2009).

17 F. Chen, M. D. Ding, and P. F. Chen, Astrophys. J. 720, 1254 (2010).

18 Y. Dai, F. Auchére, J. C. Vial, Y. H. Tang, and W. G. Zong, Astrophys. J. 708, 913 (2010).

19 W. Liu, N. V. Nitta, C. J. Schrijver, A. M. Title, and T. D. Tarbell, Astrophys. J. 723, L53 (2010).

20 H. Q. Yang, and P. F. Chen, Sol. Phys. 266, 59 (2010).

21 Y. Zhang, R. Kitai, N. Narukage, T. Matsumoto, S. Ueno, K. Shibata, and J. Wang, Publ. Astron. Soc. Jpn. 63, 685 (2011).

22 Y. Dai, M. D. Ding, P. F. Chen, and J. Zhang, Astrophys. J. 759, 55 (2012). 
23 T. Li, J. Zhang, S. Yang, and W. Liu, Astrophys. J. 746, 13 (2012).

24 T. Li, J. Zhang, S. H. Yang, and W. Liu, Res. Astron. Astrophys. 12, 104 (2012).

25 Y. Shen, and Y. Liu, Astrophys. J. 752, L23 (2012).

26 R. Zheng, Y. Jiang, J. Yang, Y. Bi, J. Hong, B. Yang, and D. Yang, Astrophys. J. 747, 67 (2012).

27 Y. Shen, Y. Liu, J. Su, H. Li, R. Zhao, Z. Tian, K. Ichimoto, and K. Shibata, Astrophys. J. 773, L33 (2013).

28 J. X. Wang, and H. S. Ji, Sci. China Earth Sci. 56, 1091 (2013).

29 Z. K. Xue, Z. Q. Qu, X. L. Yan, L. Zhao, and L. Ma, Astron. Astrophys. 556, A152 (2013).

30 L. Yang, J. Zhang, W. Liu, T. Li, and Y. Shen, Astrophys. J. 775, 39 (2013).

31 R. Zheng, Y. Jiang, J. Yang, Y. Bi, J. Hong, B. Yang, and D. Yang, Astrophys. J. 764, 70 (2013).

32 Y. Guo, M. D. Ding, and P. F. Chen, Astrophys. J. Suppl. 219, 36 (2015).

33 W. Zong, and Y. Dai, Astrophys. J. 809, 151 (2015).

34 X. P. Zhou, and H. F. Liang, Acta Astron. Sin. 57, 282 (2016).

35 P. F. Chen, S. T. Wu, K. Shibata, and C. Fang, Astrophys. J. 572, L99 (2002).

36 B. Li, H. Zheng, and S. Wang, Chin. Astron. Astrophys. 26, 458 (2002).

37 P. F. Chen, C. Fang, and K. Shibata, Astrophys. J. 622, 1202 (2005).

38 H. N. Zheng, Y. Y. Zhang, S. Wang, C. B. Wang, and Y. Li, Chin. Phys. Lett. 23, 399 (2006).

39 H. Wang, C. Shen, and J. Lin, Astrophys. J. 700, 1716 (2009).

40 X. H. Zhao, S. T. Wu, A. H. Wang, A. Vourlidas, X. S. Feng, and C. W. Jiang, Astrophys. J. 742, 131 (2011).
41 L. Zhang, H. Zheng, and C. Liao, Sci. China-Phys. Mech. Astron. 55, 1316 (2012).

42 L. Zhang, H. Zheng, and C. Liao, Chin. Astron. Astrophys. 38, 56 (2014).

43 B. J. Thompson, J. B. Gurman, W. M. Neupert, J. S. Newmark, J. P. Delaboudiniére, O. C. S. Cyr, S. Stezelberger, K. P. Dere, R. A. Howard, and D. J. Michels, Astrophys. J. 517, L151 (1999).

44 Y. M. Wang, Astrophys. J. 543, L89 (2000).

45 S. T. Wu, H. Zheng, S. Wang, B. J. Thompson, S. P. Plunkett, X. P. Zhao, and M. Dryer, J. Geophys. Res. 106, 25089 (2001).

46 C. Delannee, Astrophys. J. 545, 512 (2000).

47 P. F. Chen, and Y. Wu, Astrophys. J. 732, L20 (2011).

48 A. Warmuth, and G. Mann, Astron. Astrophys. 532, A151 (2011).

49 A. Asai, T. T. Ishii, H. Isobe, R. Kitai, K. Ichimoto, S. UeNo, S. Nagata, S. Morita, K. Nishida, D. Shiota, A. Oi, M. Akioka, and K. Shibata, Astrophys. J. 745, L18 (2012).

50 X. Cheng, J. Zhang, O. Olmedo, A. Vourlidas, M. D. Ding, and Y. Liu, Astrophys. J. 745, L5 (2012).

51 S. M. White, K. Balasubramaniam, and E. Cliver, Direct Comparison of a Solar Moreton Wave, EUV Wave and CME, Technical Report (Air Force Research Laboratory, 2013), 22, pp. 1-22.

52 P. F. Chen, Washington DC Am. Geophys. Union Geophys. Monogr. Ser. 216, 381 (2016).

53 P. F. Chen, and C. Fang, A converging view on EIT waves, in EAS Publications Series, edited by M. Faurobert, C. Fang, and T. Corbard (EDP Sciences, London, 2012), 55, pp. 313-320.

54 R. Chandra, P. F. Chen, A. Fulara, A. K. Srivastava, and W. Uddin, Astrophys. J. 822, 106 (2016).

55 P. F. Chen, C. Fang, R. Chandra, and A. K. Srivastava, Sol. Phys. 291, 3195 (2016). 